\title{
Covering morphisms of topological internal groupoids
}

\author{
Osman Mucuk ${ }^{1}$ (D), H. Fulya Akız ${ }^{* 2}$ (D) \\ ${ }^{1}$ Department of Mathematics, Erciyes University, Kayseri, Turkey \\ ${ }^{2}$ Department of Mathematics, Bozok University, Yozgat, Turkey
}

\begin{abstract}
Let $X$ be a topological group with operations whose underlying space has a universal cover. Then the fundamental groupoid $\pi X$ becomes a topological internal groupoid, i.e., an internal groupoid in the category of topological groups. In this paper, we prove that the slice category Cov sTC $_{\text {S }} X$ of covering morphisms $p: \widetilde{X} \rightarrow X$ of topological groups with operations in which $\widetilde{X}$ has also a universal cover and the category $\operatorname{Cov}_{\mathrm{Gpd}(\mathrm{TC})} / \pi X$ of covering morphisms $q$ : $\widetilde{G} \rightarrow \pi X$ of topological internal groupoids based on $\pi X$ are equivalent. We also prove that for a topological internal groupoid $G$, the category $\operatorname{Cov}_{\mathrm{Gpd}(\mathrm{TC})} / G$ of covering morphisms of topological internal groupoids based on $G$ and the category $\operatorname{Act}_{G p d(T C)} / G$ of topological internal groupoid actions of $G$ on topological groups with operations are equivalent.
\end{abstract}

Mathematics Subject Classification (2010). 18D35, 20L05, 22A22

Keywords. covering groupoid, internal groupoids, topological internal groupoids

\section{Introduction}

In the theory of covering groupoids there is a classical equivalence of categories for a groupoid $G$ of covering morphisms of the groupoid $G$ and actions of the groupoid $G$ on sets. In [10, Theorem 2] it was proved that for a topological groupoid $G$, the category $\operatorname{Cov}_{\mathrm{TGd}} / G$ of topological groupoid coverings of $G$ and the category $\operatorname{Act}_{\mathrm{TGd}}(G)$ of topological actions of $G$ are equivalent.

On the other hand there are two important results on group-groupoids, which are internal groupoids in the category of groups. One is that if $X$ is a topological group whose topology has a universal cover, then the category $\operatorname{Cov}_{\mathrm{TG}} / \mathrm{X}$ of covering groups of $X$ and the category $\operatorname{Cov}_{\mathrm{GrGd}} / \pi X$ of group-groupoid covers of $\pi X$ are equivalent [8, Proposition 2.3]. The other is that if $G$ is a group-groupoid, then the category $\operatorname{Cov}_{G r G d} / G$ of groupgroupoid covers of $G$ is equivalent to the category $\operatorname{Act}_{\mathrm{GpGd}}(G)$ of group-groupoid actions of $G$ on groups [8, Proposition 3.1]. The former equivalence is also strengthened in the local case of topological groups in [1]. These two results in [8] recently have been generalized in [2] to a wide class of algebraic structures, which include groups, rings without identity, Rmodules, Lie algebras, Jordan algebras, and many others. These are conveniently handled

\footnotetext{
*Corresponding Author.

Email addresses: mucuk@erciyes.edu.tr (O. Mucuk), fulya.gencel@bozok.edu.tr (H.F. Akı)

Received: 05.10.2018; Accepted: 24.06.2019
} 
by working in a category $\mathrm{C}$ of groups with operations in the sense of Orzech [22]. Mucuk and Şahan in [19] give the relations between the coverings of an internal groupoid within topological groups with operations and associated crossed module.

In this paper we deal with the topological cases of the results in [2] working in the category TC of topological groups with operations. So for the first one we prove that for a topological group with operations $X$ whose underlying space has a universal cover, the category Covstc/ $X$ of covering morphisms $p: \widetilde{X} \rightarrow X$ of topological group with operations in which $\tilde{X}$ has also a universal cover, is equal to the category $\operatorname{Cov}_{\mathrm{Gpd}(\mathrm{TC})} / \pi X$ of covering morphisms $q: \widetilde{G} \rightarrow \pi X$ of topological internal groupoids in which the space $\mathrm{Ob}(\widetilde{G})$ has a universal covering. For the second one we prove that if $G$ is a topological internal groupoid, then the category $\operatorname{Cov}_{\mathrm{Gpd}(\mathrm{TC})} / G$ of covering morphisms of topological internal groupoid $G$ is equivalent to the category $\operatorname{Act}_{\mathrm{Gpd}(\mathrm{TC})} / G$ of topological internal groupoid actions of $G$ on topological groups with operations.

\section{Preliminaries on groupoids and coverings}

A groupoid is a (small) category in which each morphism is an isomorphism [5, p.205]. So a groupoid $G$ has a set $G$ of morphisms, which we call just elements of $G$, a set $\mathrm{Ob}(G)$ of objects together with maps $s, t: G \rightarrow \mathrm{Ob}(G)$ and $\epsilon: \operatorname{Ob}(G) \rightarrow G$ such that $s \epsilon=t \epsilon=1_{\mathrm{Ob}(G)}$. The maps $s, t$ are called initial and final point maps respectively and the map $\epsilon$ is called object inclusion. If $a, b \in G$ and $t(a)=s(b)$, then the composite $a \circ b$ exists such that $s(a \circ b)=s(a)$ and $t(a \circ b)=t(b)$. So there exists a partial composition defined by $G_{t} \times{ }_{s} G \rightarrow G,(a, b) \mapsto a \circ b$, where $G_{t} \times{ }_{s} G$ is the pullback of $t$ and $s$. Further, this partial composition is associative, for $x \in \operatorname{Ob}(G)$ the element $\epsilon(x)$ denoted by $1_{x}$ acts as the identity, and each element $a$ has an inverse $a^{-1}$ such that $s\left(a^{-1}\right)=t(a)$, $t\left(a^{-1}\right)=s(a), a \circ a^{-1}=(\epsilon s)(a), a^{-1} \circ a=(\epsilon t)(a)$. The map $G \rightarrow G, a \mapsto a^{-1}$ is called the inversion.

In a groupoid $G$ for $x, y \in \operatorname{Ob}(G)$ we write $G(x, y)$ for the set of all morphisms with initial point $x$ and final point $y$. We say $G$ is transitive if for all $x, y \in \operatorname{Ob}(G)$, the set $G(x, y)$ is not empty. For $x \in \mathrm{Ob}(G)$ we denote the star $\{a \in G \mid s(a)=x\}$ of $x$ by $G_{x}$.

Let $p: \widetilde{G} \rightarrow G$ be a morphism of groupoids. Then $p$ is called a covering morphism and $\widetilde{G}$ a covering groupoid of $G$ if for each $\widetilde{x} \in \mathrm{Ob}(\widetilde{G})$ the restriction $\widetilde{G}_{\widetilde{x}} \rightarrow G_{p(\widetilde{x})}$ is bijective.

Assume that $p: \widetilde{G} \rightarrow G$ is a covering morphism. Then we have a lifting function $S_{f}: G_{s} \times \mathrm{Ob}(p) \mathrm{Ob}(\widetilde{G}) \rightarrow \widetilde{G}$ assigning to the pair $(a, x)$ in the pullback $G_{s} \times{ }_{\mathrm{Ob}(p)} \mathrm{Ob}(\widetilde{G})$ the unique element $b$ of $\widetilde{G}_{x}$ such that $f(b)=a$. Clearly $S_{f}$ is inverse to $(p, s): \widetilde{G} \rightarrow$ $G_{s} \times \mathrm{Ob}(p) \mathrm{Ob}(\widetilde{G})$. So it is stated that $p: \widetilde{G} \rightarrow G$ is a covering morphism if and only if $(p, s)$ is bijective.

A covering morphism $p: \widetilde{G} \rightarrow G$ is called transitive if both $\widetilde{G}$ and $G$ are transitive. A transitive covering morphism $p: \widetilde{G} \rightarrow G$ is called universal if $\widetilde{G}$ covers every cover of $G$, i.e., if for every covering morphism $q: \widetilde{H} \rightarrow G$ there is a unique morphism of groupoids $\widetilde{p}: \widetilde{G} \rightarrow \widetilde{H}$ such that $q \widetilde{p}=p$ (and hence $\widetilde{p}$ is also a covering morphism), this is equivalent to that for $\widetilde{x}, \widetilde{y} \in O_{\widetilde{G}}$ the set $\widetilde{G}(\widetilde{x}, \widetilde{y})$ has not more than one element.

A morphism $p:(\widetilde{G}, \widetilde{x}) \rightarrow(G, x)$ of pointed groupoids is called a covering morphism if the morphism $p: \widetilde{G} \rightarrow G$ is a covering morphism.

It was proved in that $[5,10.6 .1]$ that If $X$ is a topological space which has a universal cover, then the category $\operatorname{Cov}_{\text {Top }} / \mathrm{X}$ of covering maps $p: \widetilde{X} \rightarrow X$ is equivalent to the category $\operatorname{Cov}_{\mathrm{Gd}} / \pi_{1} X$ of covering morphisms $q: \widetilde{G} \rightarrow \pi_{1} X$ of groupoids.

Let $G$ be a groupoid. An action of $G$ on a set consists of a set $X$, a function $\theta: X \rightarrow$ $\operatorname{Ob}(G)$ and a function $\varphi: X_{\theta} \times{ }_{s} G \rightarrow X,(x, a) \mapsto x a$ defined on the pullback $X_{\theta} \times{ }_{s} G$ of $\theta$ and $s$ such that 
(1) $\theta(x a)=t(a)$ for $(x, a) \in X_{\theta} \times{ }_{s} G$;

(2) $x(a b)=(x a) b$ for $(a, b) \in G_{t} \times{ }_{s} G$ and $(x, a) \in X_{\theta} \times{ }_{s} G$;

(3) $x 1_{\theta(x)}=x$ for $x \in X$.

Given such an action, semidirect product groupoid $G \ltimes X$ is defined to be the groupoid with object set $X$ and elements of $(G \ltimes X)(x, y)$ the pairs $(a, x)$ such that $a \in G(\theta(x), \theta(y))$ and $x a=y$. The groupoid composite is defined to be

$$
(a, x) \circ(b, y)=(a b, x)
$$

when $y=x a$

A topological groupoid is a groupoid such that the sets $G$ of morphisms and the set $\mathrm{Ob}(G)$ of objects are topological spaces and source, target, inclusion, inverse and product maps are continuous (see for example [15, Section 2] and [7]). Let $G$ and $\widetilde{G}$ be two topological groupoids. Then a morphism $p: \widetilde{G} \rightarrow G$ of topological groupoids is a morphism of groupoids such that $p: \widetilde{G} \rightarrow G$ and $\mathrm{Ob}(p): \mathrm{Ob}(\widetilde{G}) \rightarrow \mathrm{Ob}(G)$ are continuous.

Definition 2.1. A morphism $p: \widetilde{G} \rightarrow G$ of topological groupoids is called covering morphism of topological groupoids if for each $x \in \mathrm{Ob}(\widetilde{G})$ the restriction $p_{\widetilde{x}}: \widetilde{G}_{\widetilde{x}} \rightarrow G_{p(\widetilde{x})}$ of $p$ is a homeomorphism.

Example 2.2 ([10, Example 3]). If $p: \widetilde{X} \rightarrow X$ be a covering morphism of topological groups in which both underlying spaces of $X$ and $\tilde{X}$ have universal covers, then $\pi(p): \pi \widetilde{X} \rightarrow \pi X$ becomes a covering morphism of topological groupoids.

As it is stated in [12, Section 2], equivalently a covering morphism of topological groupoids can be restated as follows:

Theorem 2.3. Let $p: \widetilde{G} \rightarrow G$ be a morphism of topological groupoid groupoids. Then $p$ is a covering morphism of topological groupoids if and only if

$$
(p, s): \widetilde{G} \rightarrow G_{s} \times \mathrm{Ob}(p) \operatorname{Ob}(\widetilde{G})
$$

is a homeomorphism.

The topological action of a topological groupoid on a topological space is defined in $[10$, p.144] as follow:

Let $G$ be a topological groupoid, $X$ a toplogical space and $G \ltimes X$ a groupoid action on $X$. If the functions $\theta: X \rightarrow \operatorname{Ob}(G)$ and $\varphi: X_{\theta} \times_{s} G \rightarrow X,(x, a) \mapsto x a$ are continuous, then we say that $G$ acts as topologically on $X$.

Example 2.4 ([10, Theorem 2]). Let $p: \widetilde{G} \rightarrow G$ be a covering morphism of topological groupoids. Then $G$ acts as topologically on $\operatorname{Ob}(\widetilde{G})$.

Recall that a covering map $p: \widetilde{X} \rightarrow X$ of connected spaces is called universal if it covers every covering of $X$ in the sense that if $q: \tilde{Y} \rightarrow X$ is another covering of $X$ then there exists a map $r: \widetilde{X} \rightarrow \widetilde{Y}$ such that $p=q r$ (hence $r$ becomes a covering). A covering map $p: \widetilde{X} \rightarrow X$ is called simply connected if $\widetilde{X}$ is simply connected. So a simply connected covering is a universal covering.

The following result was proved in [6, Theorem 1] and we need it in the proof of Theorem 3.11 .

Proposition 2.5. If $X$ is a locally path connected and semi-locally simply connected space, then the fundamental groupoid $\pi X$ may be given a topology making it a topological groupoid. 


\section{Topological groups with operations and topological internal categories}

The idea of the definition of categories of groups with operations comes from Higgins [13] and Orzech [22]; and the definition below is from Porter [23, Section 1] and Datuashvili [11, p.21], which is adapted from Orzech[22].

Definition 3.1. The notion of a group with a set of operations consists of a pair $(\Omega, E)$ where $\mathrm{E}$ is a set of identities including the group laws and $\Omega$ of operations which includes the group operations, and the following conditions hold: If $\Omega_{i}$ is the set of $i$-ary operations in $\Omega$, then

(a) $\Omega=\Omega_{0} \cup \Omega_{1} \cup \Omega_{2}$;

(b) The group operations written additively $0,-$ and + are the elements of $\Omega_{0}, \Omega_{1}$ and $\Omega_{2}$ respectively. Let $\Omega_{2}^{\prime}=\Omega_{2} \backslash\{+\}, \Omega_{1}^{\prime}=\Omega_{1} \backslash\{-\}$ and assume that if $\star \in \Omega_{2}^{\prime}$, then $\star^{\circ}$ defined by $a \star^{\circ} b=b \star a$ is also in $\Omega_{2}^{\prime}$. Also assume that $\Omega_{0}=\{0\}$;

(c) For each $\star \in \Omega_{2}^{\prime}$, E includes the identity $a \star(b+c)=a \star b+a \star c$;

(d) For each $\omega \in \Omega_{1}^{\prime}$ and $\star \in \Omega_{2}^{\prime}$, E includes the identities $\omega(a+b)=\omega(a)+\omega(b)$ and $\omega(a) \star b=\omega(a \star b)$.

From now on $C$ will be a category of groups with operations satisfying the conditions (a)-(d).

A morphism $f:(E, \Omega) \rightarrow\left(E^{\prime}, \Omega\right)$ consists of a function $f: E \rightarrow E^{\prime}$ is a group homomorphism, which preserves the operations.

Remark 3.2. The set $\Omega_{0}$ contains exactly one element, the group identity; hence for instance the category of associative rings with unit is not a category of groups with operations.

Example 3.3. The categories of groups, rings generally without identity, R-modules, associative, associative commutative, Lie, Leibniz, alternative algebras are examples of categories of groups with operations.

The category of topological groups with operations are defined in [2] as follows:

Definition 3.4. A category TC of topological groups with a set $\Omega$ of continuous operations and with a set $E$ of identities such that $E$ includes the group laws such that the conditions (a)-(d) of Definition 3.1 are satisfied, is called a category of topological groups with operations.

In the rest of the paper TC will denote the category of topological groups.

A morphism between any two objects of TC is a continuous group homomorphism, which preserves the operations in $\Omega_{1}^{\prime}$ and $\Omega_{2}^{\prime}$.

The categories of topological groups, topological rings and topological R-modules are examples of categories of topological groups with operations.

Definition 3.5 ([23, Section 3]). Let $C$ be the category of groups with operations. An internal category in $C$ is a category $C$ in which $\mathrm{Ob}(C)$ and $C$ are the objects of $C$ such that the initial and final point maps $s, t: C \rightrightarrows \mathrm{Ob}(C)$, the object inclusion map $\epsilon: \mathrm{Ob}(C) \rightarrow C$ and the partial composition $\circ: C_{t} \times{ }_{s} C \rightarrow C,(a, b) \mapsto a \circ b$ are morphisms in the category C.

Note that since $\epsilon$ is a morphism in C, $\epsilon(0)=0$ and that the operation $\circ$ being a morphism implies that for all $a, b, c, d \in C$ and $\star \in \Omega_{2}$,

$$
(a \star c) \circ(b \star d)=(a \circ b) \star(c \circ d)
$$

whenever one side makes sense. This is called the interchange law [23] .

We also note from [23] that any internal category in $C$ is an internal groupoid since, given $a \in C, a^{-1}=\epsilon t(a)-a+\epsilon s(a)$ satisfies $a^{-1} \circ a=\epsilon t(a)$ and $a \circ a^{-1}=\epsilon s(a)$. So we 
use the term internal groupoid rather than internal category and write $G$ for an internal groupoid. For the category of internal groupoids in $\mathrm{C}$ we use the notation $\mathrm{Gpd}(\mathrm{C})$. Here a morphism $f: H \rightarrow G$ in $\operatorname{Gpd}(\mathrm{C})$ is the morphism of underlying groupoids and a morphism in $\mathrm{C}$.

In particular if $\mathrm{C}$ is the category of groups, then an internal groupoid $G$ in $\mathrm{C}$ becomes a group object in the category of groupoids, which is quite often called 2-group, see for example [4], group-groupoid or G-groupoid [9, p.297], [16]. The advantages of the group-groupoid term are: (i) there is no confusion with the group theoretic term "2group" as a group all of whose elements are of order 2; (ii) it is a clear statement of the structure involved. Recently the notion of monodromy for topological group-groupoids was developed in [21, Theorem 3.10]; and the normality and quotient in group-groupoids were developed in [20]. In the case where $C$ is the category of rings, an internal groupoid is a ring object in the category of groupoids [17, Definition 1.1] (see also [3, Definition 4.1] for topological R-module case).

Example 3.6 ([2]). Let $X$ be an object of TC. Then the groupoid $G=X \times X$ is an internal groupoid.Here a pair $(x, y)$ is a morphism from $x$ to $y$ with inverse morphism $(y, x)$. The groupoid composition is defined by $(x, y) \circ(u, z)=(x, z)$ whenever $y=u$. The binary operations in $G$ are defined by $(x, y) \widetilde{\star}(u, v)=(x \star u, y \star v)$ for $\star \in \Omega_{2}$ and the unary operations by $\widetilde{\omega}(x, y)=(\omega(x), \omega(y))$ for $\omega \in \Omega_{1}$. For the interchange law if $a=(x, y), b=(y, z), c=(u, v)$ and $d=(v, w)$ are the morphisms in $G$ so that the compositions $a \circ b$ and $c \circ d$ are defined, then we have $(a \circ b) \widetilde{\star}(c \circ d)=(x \star u, z \star w)$ and $(a \widetilde{\star} c) \circ(b \widetilde{\star} d)=(x \star u, z \star w)$ and therefore we have the interchange law

$$
(a \widetilde{\star} c) \circ(b \widetilde{\star} d)=(a \circ b) \widetilde{\star}(c \circ d) .
$$

Remark 3.7 ([2, 3.7]). The following are immediate from Definition 3.5:

(i) By Definition 3.5 we know that in an internal groupoid $G$ in $\mathrm{C}$, the initial and final point maps $s$ and $t$, the object inclusion map $\epsilon$ are the morphisms in $C$ and the interchange law (3.1) is satisfied. Therefore in an internal groupoid $G$, the unary operations are endomorphisms of the underlying groupoid of $G$ and the binary operations are morphisms from the underlying groupoid of $G \times G$ to the one of $G$.

(ii) Let $G$ be an internal groupoid in $\mathrm{C}$ and $0 \in \mathrm{Ob}(G)$ the identity element. Then Kers $=\mathrm{St}_{G} 0$, called in [5, p.3] transitivity component or connected component of 0 , is also an internal groupoid.

Let $H$ and $G$ be two internal groupoids in C. A morphism of internal groupoids is a morphism $f: H \rightarrow G$ of underlying groupoids which is also a morphism of groups with operations. A morphism $f: H \rightarrow G$ of internal groupoids is called covering (resp. universal covering) if it is a covering (resp. universal covering) morphism on the underlying groupoids.

Definition 3.8. An internal groupoid in the category TC of topological groups with operations is called a topological internal groupoid.

So a topological internal groupoid is a topological groupoid $G$ in which the set of morphisms and the set $\mathrm{Ob}(G)$ of objects are objects of TC and all structural maps of $G$, i.e, the source and target maps $s, t: G \rightarrow \operatorname{Ob}(G)$, the object inclusion map $\epsilon: \operatorname{Ob}(G) \rightarrow G$ and the composition map o: $G_{t} \times{ }_{s} G \rightarrow G$, are morphisms of TC.

If TC is the category of topological groups, then an internal topological groupoid becomes a topological group-groupoid which was previously defined in [14, Definition 1] by the requirement that is a topological groupoid which is also a group-groupoid with continuous structural maps of groups.

Example 3.9. A topological group with operations which is abelian according to all binary operations $\star \in \Omega_{2}$ can be thought as a topological internal groupoid. 
Example 3.10 ([18, Example 3.10]). Let $X$ be an object of TC. Then the groupoid $G=X \times X$ is a topological internal groupoid. By Example 3.6 it is seen that $G=X \times X$ is an internal groupoid. In addition since the maps $s, t, \epsilon$ and the groupoid composition $\circ: G_{t} \times{ }_{s} G \rightarrow G,(a, b) \mapsto a \circ b$ are continuous then the structural maps of $G$ are continuous. Hence $G$ becomes a topological internal groupoid.

Theorem 3.11. Let $X$ be an object of TC such that the underlying space is locally path connected and semi-locally simply connected. Then the fundamental groupoid $\pi X$ is a topological internal groupoid.

Proof. Let $X$ be a topological group with operations as assumed. By Theorem 2.5, $\pi X$ has a topology such that it is a topological groupoid. We know by [6, Proposition 3] that when $X$ and $Y$ are endowed with such topologies, for a continuous map $f: X \rightarrow Y$, the induced morphism $\pi(f): \pi X \rightarrow \pi Y$ is also continuous. Hence the continuous binary operations $\star: X \times X \rightarrow X$ for $\star \in \Omega_{2}$ and the unary operations $\omega: X \rightarrow X$ for $\omega \in \Omega_{1}$ respectively induce continuous binary operations $\widetilde{\star}: \pi X \times \pi X \rightarrow \pi X$ and unary operations $\widetilde{\omega}: \pi X \rightarrow \pi X$. So the set of morphisms becomes a topological group with operations. Now we show that the groupoid structural maps are morphisms of groups with operations, i.e., preserve the operations.

For the morphism $s: \pi X \rightarrow X, s([a])=a(0)$, the operations $\star \in \Omega_{2}$ and $\omega \in \Omega_{1}$ we have the following:

$$
\begin{aligned}
s([a \star b]) & =(a \star b)(0) \\
& =a(0) \star b(0) \\
& =s[a] \star s[b]
\end{aligned}
$$

and

$$
\begin{aligned}
s(\omega[a]) & =s[\omega(a)] \\
& =(w(a))(0) \\
& =w(a(0)) \\
& =w s[a] .
\end{aligned}
$$

For the final map $t: \pi X \rightarrow X, t([a])=a(1)$, the operations $\star \in \Omega_{2}, \omega \in \Omega_{1}$ and for $[x] \in \pi X$ the following are satisfied:

$$
\begin{aligned}
t([a \star b]) & =(a \star b)(1) \\
& =a(1) \star b(1) \\
& =t[a] \star t[b]
\end{aligned}
$$

and

$$
\begin{aligned}
t(\omega[a]) & =t[\omega(a)] \\
& =(w(a))(1) \\
& =w(a(1)) \\
& =w t[a] .
\end{aligned}
$$

Since $X$ is a topological group with operations, then for the partial composition $\pi X_{t} \times_{s}$ $\pi X \rightarrow \pi X,([x],[y]) \mapsto[x \circ y]$ and $x, y, z, t \in X$,

$$
[(x \star z) \circ(y \star t)]=[(x \circ y) \star(t \circ z)],
$$

where $(x \circ y)$ and $(t \circ z)$ are defined. This equivalence shows us that the partial composition is a morphism of groups with operations.

Therefore $\pi X$ becomes a topological internal groupoid. 
Let STC be the full subcategory of TC on those objects whose underlying spaces are locally path connected and semi-locally simply connected; and let $\mathrm{Gpd}(\mathrm{TC})$ be the category of topological internal groupoids in $\mathrm{C}$. Then we have a functor

$$
\pi: \mathrm{sTC} \rightarrow \operatorname{Gpd}(\mathrm{TC}) \text {. }
$$

\section{Covering morphisms of topological internal groupoids}

We now give the definition of a covering morphism of topological internal groupoids:

Definition 4.1. Let $\widetilde{G}$ and $G$ be topological internal groupoids and let $p$ : $\widetilde{G} \rightarrow G$ be a morphism of topological internal groupoids. Then $p$ is called a covering morphism of topological internal groupoids if it is also a covering morphism of topogical groupoids.

Example 4.2. Let $p: \widetilde{X} \rightarrow X$ be a covering morphism of topological groups with operations in which $X$ and $\widetilde{X}$ has universal covers. We know from Theorem 3.11 that $\pi \widetilde{X}$ and $\pi X$ are topological internal groupoids. Then the induced morphism $\pi(p): \pi \widetilde{X} \rightarrow \pi X$ is a covering morphism of topological internal groupoids in $\mathrm{C}$.

Let $X$ be a topological group with operations which has a universal cover. Let $\operatorname{Cov}_{\mathbf{s} T C} / X$ be the category of covering morphisms $p: \widetilde{X} \rightarrow X$ of topological groups with operations in which $\tilde{X}$ has also a universal cover. Let $\operatorname{Cov}_{\mathrm{Gpd}(\mathrm{TC})} / \pi X$ be the category of covering morphisms $p: \widetilde{G} \rightarrow \pi X$ of topological internal groupoids based on $\pi X$ such that $\operatorname{Ob}(\widetilde{G})$ has a universal cover. We now prove the following equivalence of the categories.

Theorem 4.3. The categories $\operatorname{Cov}_{\mathrm{sTC}} / X$ and $\operatorname{Cov}_{\mathrm{Gpd}(\mathrm{TC})} / \pi X$ are equivalent.

Proof. Define a functor

$$
\pi: \operatorname{Cov}_{\mathrm{sTC}} / X \rightarrow \operatorname{Cov}_{\mathrm{Gpd}(\mathrm{TC})} / \pi X
$$

as follows: Suppose that $p: \tilde{X} \rightarrow X$ is a covering morphism of topological groups with operations in which both underlying spaces $X$ and $\widetilde{X}$ have also a universal covers. From $[2$, Theorem 3.1] the induced morphism $\pi(p): \pi \widetilde{X} \rightarrow \pi X$ is a morphism of internal groupoids and a covering morphism on the underlying groupoids. By Theorem 3.11, $\pi X$ and $\pi \widetilde{X}$ are topological internal groupoids. Since the map $(\pi(p), s)$ in the sense of Theorem 2.3 is a homeomorphism and so $\pi(p)$ is a covering morphism of topological groupoids [10, Example 3]. Therefore $\pi(p): \pi \widetilde{X} \rightarrow \pi X$ is a covering morphism of topological internal groupoids. In this way we have a functor as required.

We now define a functor

$$
\Gamma: \operatorname{Cov}_{\mathrm{Gpd}(\mathrm{TC})} / \pi_{1} X \rightarrow \operatorname{Cov}_{\mathrm{sTC}} / X
$$

as follows: Let $q: \widetilde{G} \rightarrow \pi_{1} X$ be a covering morphism of topological internal groupoids in which $\operatorname{Ob}(\widetilde{G})=\widetilde{X}$ and $X$ have a universal cover. By the lifted topology on $\widetilde{X}[5,10.5 .5]$ we have a covering morphism $p: \widetilde{X} \rightarrow X$ of topological spaces where $p=\operatorname{Ob}(q)$ and $\widetilde{X}=\operatorname{Ob}(\widetilde{G})$. Since $q$ is a covering morphism of topological internal groupoid, $q$ and $\mathrm{Ob}(q)=p$ are morphisms of topological groups with operations.

Since the category $\operatorname{Cov}_{\text {Top }} / X$ of topological covering maps of $X$ is equivalent to the category $\operatorname{Cov}_{\mathrm{Gd}} / \pi(X)$ of groupoid covering morphisms of $\pi X$, by the following diagram the proof is completed.

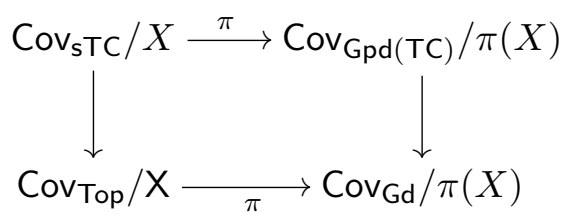


We define an action of a topological internal groupoid on a topological group with operation as follows:

Definition 4.4. Let $G$ be a topological internal groupoid in TC and $X$ an object of TC. If the underlying groupoid of $G$ acts on the underlying set of $X$ such that $\theta: X \rightarrow \operatorname{Ob}(G)$ and $\varphi: X_{\theta} \times{ }_{s} G \rightarrow X,(x, a) \mapsto x a$ are morphisms in TC, then we say that the topological internal groupoid $G$ acts as topologically on the topological group with operations $X$ via $\theta$.

We write $(X, \theta, \varphi)$ for an action.

Here note that $\varphi: X_{\theta} \times{ }_{s} G \rightarrow X,(x, a) \mapsto x a$ is a morphism in TC, then we have

$$
(x \star y)(a \star b)=(x a) \star(y b)
$$

for $x, y \in X$ and $a, b \in G$ whenever one side is defined.

Example 4.5. Let $G$ and $\widetilde{G}$ be topological internal groupoids in TC and let $p: \widetilde{G} \rightarrow G$ be a covering morphism of of topological internal groupoids as defined in Definition 4.1. Then the topological internal groupoid $G$ acts topologically on the topological group with operations $\widetilde{X}=\operatorname{Ob}(\widetilde{G})$ via $\operatorname{Ob}(p)$ assigning to $\widetilde{x} \in \widetilde{X}$ and $a \in G_{p(\widetilde{x})}$ the target of the unique lifting of $a$ with source $\widetilde{x}$ : In fact $p: \widetilde{G} \rightarrow G$ is a covering morphism of topological groupoids and therefore by Example 2.4 the topological groupoid $G$ act topologically on the space $\widetilde{X}=\mathrm{Ob}(\widetilde{G})$ via $\mathrm{Ob}(p)$. The condition

$$
(\widetilde{x} \star \widetilde{y})(a \star b)=(\widetilde{x} a) \star(\widetilde{y} b)
$$

is satisfied for $\widetilde{x}, \widetilde{y} \in \widetilde{X}$ and $a, b \in G$ whenever one sight is defined. The other conditions related to the group operations are satisfied.

We know from [10, p.145] that if $G$ is a topological groupoid and $X$ is a topological space such that $G$ acts topologically on $X$, then the semidirect product groupoid $G \ltimes X$ becomes a topological groupoid. The internal groupoid case of the following theorem was given in [2, Theorem 2.15]. So we obtain the following result for topological internal groupoids.

Theorem 4.6. Let $G$ be a topological internal category in TC and $X$ an object of TC. Suppose that the topological internal groupoid $G$ acts as topologically on the topological group with operations $X$. Then the semidirect product groupoid $G \ltimes X$ becomes a topological internal groupoid in TC such that the projection $p: G \ltimes X \rightarrow G$ is a morphism of topological internal groupoids.

Let $G$ be a topological internal groupoid in the category TC of topological groups with operations. Then we have a category $\operatorname{Cov}_{\mathrm{Gpd}(\mathrm{TC})} / G$ of covering morphisms of topological internal groupoids based on $G$. So the objects are the covering morphisms $p: \widetilde{G} \rightarrow G$ of topological internal groupoids and a morphism say from $p: \widetilde{G} \rightarrow G$ to $q: \widetilde{H} \rightarrow G$ is a morphism $f: \widetilde{G} \rightarrow \widetilde{H}$ of topological internal groupoids which becomes also a covering morphism, such that $q f=p$.

Let $\operatorname{Act}_{\mathrm{Gpd}(\mathrm{TC})}(G)$ be the category of topological internal groupoid actions on topological groups with operations. So an object of $\operatorname{Act}_{\mathrm{Gpd}(\mathrm{TC})}(G)$ is an topological internal groupoid action $(X, \theta, \varphi)$ of $G$ on a topological group with operations $X$ and a morphism say from $(X, \theta, \varphi)$ to $(X, \theta, \varphi)$ is a morphism of topological groups with operations $f: X \rightarrow X^{\prime}$ such that $\theta=\theta^{\prime} f$ and $f(x a)=(f x) a$ whenever $x a$ is defined.

In [10, Theorem 2] it was proved that for a topological groupoid $G$, the category $\operatorname{Act}_{\mathrm{TGd}}(G)$ of topological groupoid actions of $G$ and the category CovTGd/G of covering morphisms of topological groupoids based on $G$ are equivalent. We now generalize this equivalence to the topological internal groupoids as follows: 
Theorem 4.7. The categories $\operatorname{Cov}_{\mathrm{Gpd}(\mathrm{TC})} / G$ and $\mathrm{Act}_{\mathrm{Gpd}(\mathrm{TC})} / G$ are equivalent.

Proof. We define a functor

$$
\Gamma: \operatorname{Cov}_{\mathrm{Gpd}(\mathrm{TC})} / G \rightarrow \operatorname{Act}_{\mathrm{Gpd}(\mathrm{TC})}(G)
$$

as follows: Let $p: \widetilde{G} \rightarrow G$ be a covering morphism of topological internal groupoid based on $G$. Then by Example 4.5 topological internal groupoid $G$ acts as topologically on topological group with operations $\mathrm{Ob}(\widetilde{G})$ via $\theta$.

We now define a functor

$$
\Phi: \operatorname{Act}_{G \mathrm{gd}(\mathrm{TC})}(G) \rightarrow \operatorname{Cov}_{\mathrm{Gpd}(\mathrm{TC})} / G
$$

as follows: Let $(X, \theta, \varphi)$ be a topological internal groupoid action on a topological group with operation. So by [8] the projection $p: G \ltimes X \rightarrow G$ is covering morphism of topological groupoids and by [2, Theorem 3.14] is a covering morphism of internal groupoids. By compounding these we have that $p$ is a covering morphism of topological internal groupoids.

The natural equivalences $\Gamma \Phi \simeq 1$ and $\Phi \Gamma \simeq 1$ follow.

Acknowledgment. We would like to thank to the referee for useful comments and suggestions which improve the paper.

\section{References}

[1] H.F. Akız, Covering Morphisms of Local Topological Group-Groupoids, Proc. Nat. Acad. Sci. India Sect. A, 88 (4), 2018.

[2] H.F. Akız, N. Alemdar, O. Mucuk and T. Şahan, Coverings of internal groupoids and crossed modules in the category of groups with operations, Georgian Math. J. 20 (2), 223-238, 2013.

[3] N. Alemdar and O. Mucuk, The liftings of R-modules to covering groupoids, Hacet. J. Math. Stat. 41 (6), 813-822, 2012.

[4] J.C. Baez and A.D. Lauda, Higher-dimensional algebra. V. 2-groups. Theory Appl. Categ. 12 423-491, 2004.

[5] R. Brown, Topology and groupoids, Booksurge PLC, 2006.

[6] R. Brown and G. Danesh-Naruie, The Fundamental Groupoid as a Topological Groupoid, Proc. Edinb. Math. Soc. 19 (2), 237-244, 1975.

[7] R. Brown and J.P.L. Hardy, Topological Groupoids I: Universal Constructions, Math. Nachr. 71, 273-286, 1976.

[8] R. Brown and O. Mucuk, Covering groups of non-connected topological groups revisited, Math. Proc. Camb. Phill. Soc. 115, 97-110, 1994.

[9] R. Brown and C.B. Spencer , G-groupoids, crossed modules and the fundamental groupoid of a topological group, Proc. Konn. Ned. Akad. v. Wet. 79, 296-302, 1976.

[10] R. Brown, G. Danesh-Naruie and J.P.L. Hardy, Topological groupoids II: Covering morphisms and G-spaces, Math. Nachr. 74, 143-156, 1976.

[11] T. Datuashvili, Categorical, homological and homotopical properties of algebraic objects, Dissertation, Georgian Academy of Science, Tbilisi, 2006.

[12] J.P. Hardy, Topological Groupoids: Coverings and Universal Constractions, University Colloge of North., 1974.

[13] P.J. Higgins, Groups with multiple operators, Proc. London Math. Soc. 3 (6), 366-416, 1956.

[14] İ. İçen, A.F. Özcan and M.H. Gürsoy, Topological group-groupoids and their coverings, Indian J. Pure Appl. Math. 36 (9), 493-502, 2005.

[15] K.C.H. Mackenzie, Lie groupoids and Lie algebroids in differential geometry, London Math. Soc. Lecture Note Series 124, Cambridge University Press, 1987. 
[16] O. Mucuk, Covering groups of non-connected topological groups and the monodromy groupoid of a topological groupoid, $\mathrm{PhD}$ Thesis, University of Wales, 1993.

[17] O. Mucuk, Coverings and ring-groupoids, Georgian Math. J. 5, 475-482, 1998.

[18] O. Mucuk and H.F. Akız, Monodromy Groupoid of an Internal Groupoid in Topological Groups with Operations, Filomat, 29, 2355-2366, 2015.

[19] O. Mucuk and T. Şahan, Coverings and crossed modules of topological groups with operations, Turkish J. Math. 38, 833-845, 2014.

[20] O. Mucuk, T. Şahan and N. Alemdar, Normality and quotients in crossed modules and group-groupoids, Appl. Categor. Struct. 23, 415-428, 2015.

[21] O. Mucuk, B. Kıllı̧arslan, T. Şahan and N. Alemdar, Group-groupoid and monodromy groupoid, Topology Appl. 158, 2034-2042, 2011.

[22] G. Orzech, Obstruction theory in algebraic categories I and II, J. Pure. Appl. Algebra 2, 287-314 and 315-340, 1972.

[23] T. Porter, Extensions, crossed modules and internal categories in categories of groups with operations, Proc. Edinb. Math. Soc. 30, 373-381, 1987. 